\title{
O cotidiano de idosos com insuficiência venosa que usam meia elástica de compressão
}

Everyday life of elderly with venous insufficiency, who use elastic compression socks

\author{
La vida cotidiana de las personas mayores con insuficiencia venosa que usan \\ calcetines de compresión.
}

Marcelo Henrique Silva 1*, Daniele Knopp Ribeiro², Rodolfo Ribeiro de Jesus ${ }^{3}$, Renata Evangelista Tavares Machado ${ }^{4}$, Maria Cristina Pinto de Jesus², Miriam Aparecida Barbosa Merighi ${ }^{4}$

\section{ORCID IDs}

Silva MH (DD https://orcid.org/0000-0002-6250-5050 Ribeiro DK (D) http://orcid.org/0000-0002-7302-2601 Jesus RR (D) https://orcid.org/0000-0003-4606-0802 Machado RET (D) http://orcid.org/0000-0001-9004-3941 Jesus MCP (D) http://orcid.org/0000-0002-8854-690X Merighi MAB (D) http://orcid.org/0000-0002-9705-2557

\section{COMO CITAR}

Silva MH, Ribeiro DK, Jesus RR, Machado RET, Jesus MCP, Merighi MAB. O cotidiano de idosos com insuficiência venosa que usam meia elástica de compressão. ESTIMA, Braz. J. Enterostomal Ther., 17: e1519. https://doi.org/10.3086/estima.v17.736_PT

\section{RESUMO}

Objetivo: Compreender o cotidiano de idosos com insuficiência venosa que usam meia elástica de compressão. Métodos: Pesquisa fenomenológica que entrevistou 13 idosos atendidos em Unidades Básicas de Saúde no estado de Minas Gerais, entre novembro de 2017 e janeiro de 2018. Os depoimentos foram organizados em categorias e discutidos segundo o referencial da fenomenologia social de Alfred Schütz e literatura temática. Resultados: Emergiram das entrevistas as categorias que explicitam a perspectiva dos participantes sobre seu dia a dia com a meia elástica de compressão: "alívio dos principais sintomas da insuficiência venosa", "adversidades que permeiam o uso das meias elásticas de compressão" e "uso de meia elástica de compressão em longo prazo". Conclusão: Para os idosos com insuficiência venosa crônica, o uso da meia elástica de compressão promove alívio dos sintomas, porém requer ajuda para calçar e descalçar e provoca sensação de calor e desconforto nos membros inferiores. Os idosos esboçaram a expectativa de continuar usando a meia elástica em longo prazo com vistas a controlar a recidiva de úlcera varicosa. Valorizar as adversidades relacionadas ao uso desse dispositivo de compressão poderá auxiliar esses idosos a ampliar sua autonomia e capacidade funcional comprometidas pela insuficiência venosa.

DESCRITORES: Meias de compressão. Idoso. Relações interpessoais. Enfermagem. Estomaterapia. Pesquisa qualitativa.

\footnotetext{
1.Prefeitura Municipal de Juiz de Fora - Secretaria de Saúde - Departamento de Atenção Primária à Saúde - Juiz de Fora/MG - Brasil.

2.Universidade Federal de Juiz de Fora - Faculdade de Enfermagem - Departamento de Enfermagem Básica - Juiz de Fora/MG - Brasil. 3. Universidade Presidente Antônio Carlos - Faculdade de Medicina - Juiz de Fora/MG - Brasil.

4.Universidade de São Paulo - Escola de Enfermagem - Departamento de Enfermagem Materno-Infantil e Psiquiátrica - São Paulo/SP - Brasil.
}

Autor correspondente: marcelohenfermar@gmail.com

Recebido: Abr. 92019 | Aceito: Jul. 102019 


\begin{abstract}
Objective: To comprehend every day of older people with venous insufficiency, who use elastic compression socks. Methods: Phenomenological research that interviewed 13 elderly attended Basic Health Units in the state of Minas Gerais, between November 2017 to January 2018. The depositions were organized in categories and discussed according to the Alfred Schütz social phenomenology and theme literature. Results: Emerged from the interviews that make explicit the perspective of the participants regarding their everyday activities with compression elastic socks: "main symptoms of the venous insufficiency relieved", "adversities that permeate the use of elastic compression socks" and "long term use of compression elastic socks". Conclusion: For elderly people with chronic venous insufficiency, the use of elastic compression socks promotes relieve of the symptoms; however, it demands help to put them on and take them off and provokes heat sensation and discomfort in the limbs. The elderly showed the expectation to continue the long term use of the elastic sock aiming at avoiding the relapse of varicose ulcer. To validate the adversities related to the use of this compression device may help the elderly to broaden their autonomy and functional capacity, which may be compromised by venous insufficiency.
\end{abstract}

DESCRIPTORS: Compression socks. Elderly. Interpersonal relationships. Nursing. Stomatherapy. Qualitative research.

\title{
RESUMEN
}

Objetivo: Comprender la vida cotidiana de las personas mayores con insuficiencia venosa que usan calcetines de compresión. Métodos: Investigación fenomenológica que entrevistó a 13 adultos mayores atendidos en Unidades Básicas de Salud en el estado de Minas Gerais, entre noviembre de 2017 y enero de 2018. Las declaraciones fueron organizadas en categorías y discutidas de acuerdo con el marco de la fenomenología social y la literatura temática de Alfred Schütz. Resultados: De las entrevistas surgieron las categorías que explican la perspectiva de los participantes sobre su vida diaria con la calcetines de compresión: "alivio de los principales síntomas de insuficiencia venosa", "adversidades que impregnan el uso de calcetines de compresión elásticas" y "uso de calcetines de compresión a largo plazo". Conclusión: Para las personas mayores con insuficiencia venosa crónica, el uso de calcetines de compresión proporciona alivio de los síntomas, pero requiere ayuda para poner y quitar y provoca una sensación de calor e incomodidad en las extremidades inferiores. Los adultos mayores describieron la expectativa de continuar usando calcetines a largo plazo para controlar la recurrencia de la úlcera varicosa. Mejorar las adversidades relacionadas con el uso de este dispositivo de compresión puede ayudar a estos adultos mayores a aumentar su autonomía y capacidad funcional comprometida por la insuficiencia venosa.

DESCRIPTORES: Calcetines de compresión. Personas mayores. Relaciones interpersonales. Enfermería. Estomaterapia. Investigación cualitativa.

\section{INTRODUÇÃO}

A insuficiência venosa se configura como uma doença comum na população. A incidência varia de 1 a $10 \%$ na população mundial, aumentando com o avançar da idade, sendo responsável por custos significativos para a sociedade quanto ao tratamento médico e cirúrgico e, principalmente, afetando a produtividade no trabalho devido à dor e incapacidade provenientes dessa enfermidade ${ }^{1}$. O desenvolvimento da insuficiência venosa tem sido associado a múltiplos fatores predisponentes, como idade, gênero, gravidez, hereditariedade, hábitos corporais e estilo de vida. Dentre os fatores não modificáveis que aumentam a probabilidade de desenvolver varizes destacam-se o sexo e a idade ${ }^{2}$.

Define-se insuficiência venosa como o resultado da incompetência das válvulas dos sistemas venoso superficial e profundo presentes nos membros inferiores. Se não tratada adequadamente, pode levar a complicações que incluem edema, surgimento de úlceras varicosas e trombose venosa profunda, entre outros sinais e sintomas ${ }^{3}$. Diante do envelhecimento populacional progressivo, torna-se importante o desenvolvimento de ações que visem ampliar a eficácia do tratamento e a redução de complicações dessa doença.

O tratamento da insuficiência venosa envolve a utilização de medidas cirúrgicas e conservadoras. Entre as medidas conservadoras, elencam-se farmacoterapia, mudanças de estilo de vida (perda de peso, realização sistemática de exercício físico, elevação dos membros inferiores, reabilitação da articulação do tornozelo e evitação da posição de pé e/ou sentada por longos períodos), além da terapia compressiva ${ }^{3}$.

A terapia compressiva pode ser classificada de acordo com a pressão que exerce sobre o membro inferior: leve $(<20$ $\mathrm{mmHg}$ ), moderada ( $\geq 20-40 \mathrm{mmHg})$, forte $(\geq 40-60 \mathrm{mmHg}$ ) e muito forte (> $60 \mathrm{mmHg}$ ). A classificação das meias de compressão difere entre os países ${ }^{4}$. No Brasil, por exemplo, encontram-se meias com pressões que variam de 15 a 50 $\mathrm{mmHg}$, as quais podem ser indicadas, dependendo do grau de compressão, para profilaxia e tratamento da insuficiência venosa e também para tratamento e prevenção de recidiva da úlcera varicosa ${ }^{5}$. Quando devidamente indicada, reduz 
o edema e a dor e melhora os parâmetros hemodinâmicos em pacientes com insuficiência venosa, independentemente do tipo e comprimento da meia ${ }^{6}$.

Estudo que avaliou a qualidade de vida de indivíduos com doença venosa crônica usuários e não usuários de meias elásticas mostrou que o uso dessas apresentava-se como terapêutica eficaz, melhorando a qualidade de vida específica, principalmente no aspecto extensão da varicosidade, havendo também ganho na qualidade de vida geral e nos aspectos funcionais e emocionais quando comparada à dos indivíduos que não faziam uso desse dispositivo terapêutico ${ }^{7}$.

A capacidade funcional configura-se como importante preditor de saúde da pessoa idosa, e o declínio dessa caracteriza-se por perdas de habilidade para executar tarefas cotidianas que, de modo progressivo, poderão desencadear o desenvolvimento de instabilidade postural, imobilidade, entre outras ${ }^{8}$.

Considerando os benefícios da utilização assídua de meias elásticas de compressão por pessoas idosas com insuficiência venosa e o impacto positivo desse uso para manutenção da capacidade funcional, torna-se relevante compreender a experiência desse grupo populacional no que se refere ao uso desse dispositivo terapêutico. Essa oportunidade de focalizar a intersubjetividade inscrita no cotidiano desses idosos que experienciam o uso de meias elásticas de compressão vai ao encontro de propostas da Organização Mundial de Saúde (OMS) que sinalizam a relevância de cuidados em saúde centrados na pessoa idosa, baseados em suas necessidades e preferências ${ }^{9}$.

Desse modo, este estudo se norteou pelas questões: como é o cotidiano das pessoas idosas com insuficiência venosa que utilizam meias elásticas de compressão? Quais suas expectativas no tocante ao uso desse dispositivo?

\section{OBJETIVO}

Compreender o cotidiano de idosos com insuficiência venosa que usam meia elástica de compressão.

\section{MÉTODOS}

Estudo qualitativo de abordagem fenomenológica proveniente de investigação realizada no programa de iniciação científica de uma universidade pública localizada no interior do estado de Minas Gerais. Adotou-se o referencial teórico-metodológico da fenomenologia social de Alfred Schütz ${ }^{10}$ para fundamentar a compreensão do cotidiano de idosos com insuficiência venosa no que concerne ao uso de meias elásticas.

Parte-se do pressuposto de que, no mundo cotidiano, os seres humanos dotados de consciência essencialmente similar convivem uns com os outros, influenciando e sendo influenciado no que diz respeito às situações práticas da vida social. Nesse sentido, a intersubjetividade é precondição da vida cotidiana, sendo a experiência vivida fonte dos significados humanos. A pessoa interpreta o mundo a partir de sua situação biográfica e acervo de experiências prévias. Esse acervo é utilizado para a significação das atividades mundanas. Por meio de motivos existenciais: "motivos para", que orientam a ação futura, e "motivos porque", que dizem respeito à explicação posterior ao acontecimento ${ }^{10}, \mathrm{o}$ homem direciona seu agir na realidade social. Neste estudo, a ação refere-se à conduta das pessoas idosas com insuficiência venosa quanto ao uso de meias elásticas no dia a dia.

A pesquisa teve como cenário as unidades básicas de saúde de uma cidade de Minas Gerais. Nessa cidade, utiliza-se a Central de Marcação de Consultas para referenciar pacientes às especialidades médicas. Cada unidade de saúde conta com um agente administrativo e um computador que viabiliza o acesso online à central de marcação. Os encaminhamentos em formulário físico feitos pelos profissionais de saúde são arquivados pelo agente até que a marcação seja efetuada para o especialista.

O levantamento dos possíveis participantes da pesquisa foi realizado nos terminais da Central de Marcação de Consultas, por meio de busca nas fichas de encaminhamento dos pacientes ao serviço de angiologia do município. De posse da relação de pessoas atendidas pelo angiologista, os pesquisadores fizeram contato telefônico com essas pessoas e indagaram sobre a indicação de meia elástica. Nessa oportunidade, realizou-se o convite para participação no estudo, explicitando-se os objetivos e os aspectos éticos da pesquisa.

Estabeleceu-se, como critério de inclusão: idade igual ou superior a 60 anos, ambos os sexos, com diagnóstico de insuficiência venosa e indicação de uso de meias elásticas de compressão, independentemente do estágio clínico da doença e de terapêutica medicamentosa associada. Não foram incluídas as pessoas idosas que, embora tivessem prescrição de meias elásticas, não faziam uso desse dispositivo na época da pesquisa.

A coleta dos depoimentos foi realizada entre os meses de novembro de 2017 e janeiro de 2018, após autorização 
formal por meio da assinatura do Termo de Consentimento Livre e Esclarecido. Os depoimentos foram apreendidos por meio da entrevista fenomenológica norteada pelas questões: conte-me como é o seu dia a dia com as meias elásticas de compressão. Qual sua expectativa em relação ao uso de meias elásticas, considerando seu problema de varizes? Incluíram-se na entrevista informações pessoais e socioeconômicas, além do tempo de uso desse dispositivo de compressão.

Os participantes foram abordados individualmente, em sala reservada dos serviços de saúde e no domicílio, conforme sua escolha. As entrevistas tiveram a duração média de 40 minutos e o áudio foi gravado com smartphone, com permissão dos entrevistados.

O número de participantes não foi definido previamente. As entrevistas foram encerradas quando o conteúdo dos depoimentos foi considerado suficiente para o alcance dos objetivos da pesquisa. Conforme princípios da pesquisa qualitativa, foi possível aprofundar a compreensão do objeto estudado com a abrangência necessária ${ }^{11}$.

Realizaram-se 13 entrevistas, todas incluídas no estudo. Para garantir o anonimato dos participantes, os depoimentos foram identificados com a letra " $E$ " inicial da palavra "entrevista" seguida de numeração arábica correspondente à sua ordem de ocorrência (E1 a E13).

A organização e a categorização do material foram realizadas em conformidade com os pressupostos teóricometodológicos adotados em estudo fundamentado pela fenomenologia social ${ }^{12}$ : leitura criteriosa de cada depoimento para apreender o modo como pessoas idosas com insuficiência venosa experienciam o uso de meias elásticas de compressão no cotidiano; agrupamento dos aspectos significativos dos depoimentos para composição das categorias temáticas; análise e discussão dessas categorias à luz da fenomenologia social de Alfred Schütz, e outros referenciais relacionados ao tema.

$\mathrm{O}$ estudo respeitou as exigências formais contidas nas normas nacionais e internacionais regulamentadoras de pesquisas envolvendo seres humanos. $\mathrm{O}$ projeto de pesquisa foi aprovado pelo comitê de ética em pesquisa conforme o parecer 2.056.709/2017.

\section{RESULTADOS E DISCUSSÃO}

A pesquisa foi composta de 13 idosos, com idades entre 60 e 89 anos, maior representatividade do sexo feminino, ensino fundamental incompleto, aposentados e com renda familiar de um salário mínimo. O tempo de utilização de meia elástica de compressão variou de 10 a 41 anos.

O cotidiano de pessoas idosas com insuficiência venosa, quanto ao uso de meias elásticas de compressão, foi traduzido mediante as categorias temáticas: "alívio dos principais sintomas da insuficiência venosa", "adversidades que permeiam o uso das meias elásticas de compressão" " "uso de meia elástica de compressão em longo prazo". As duas primeiras categorias expressam os "motivos porque" (ações vividas no passado e presente) e a última o "motivo para" (suas expectativas relacionadas à continuidade do uso desse dispositivo).

\section{Alívio dos principais sintomas da insuficiência venosa}

Ao refletir sobre o uso das meias elásticas de compressão, as pessoas idosas relataram perceber melhora dos sintomas da insuficiência venosa: diminuição do edema e da dor nos membros inferiores, além da remissão da úlcera varicosa, permitindo o desempenho das atividades cotidianas:

"Com a meia, dói menos e dá mais firmeza na perna para andar, fazer as coisas. A ferida está melhorando depois que estou usando a meia." (E1)

"Minha perna não incha porque estou de meia. A meia não deixa a perna doer e não me prejudica em nada [...] porque não tem ninguém para fazer meu serviço, eu tenho que fazer." (E3)

"Eu me senti bem com a meia, parece que a perna ficou leve, apertou as veias e até sarou o machucado na perna [...] estou livre para fazer minhas coisas.” (E5)

"Melhorei muito mesmo, as pernas inchavam, ficavam avermelhadas e doía muito. Depois que passei a usar a meia, melhorei as dores nas pernas. Antes eu não tinha posição de ficar, agora consigo andar.” (E7)

A experiência positiva sobre o uso de meias elásticas de compressão é percebida quando a pessoa idosa começa a identificar a melhora dos sintomas da insuficiência venosa crônica e retoma minimamente suas atividades diárias. Essa experiência deve ser valorizada pelos profissionais de saúde, incluindo enfermeiros, com vistas a ampliar o uso dessa modalidade de terapia compressiva entre idosos. 
Estudo realizado nos Países Baixos revelou que adultos e idosos que utilizaram meias elásticas de compressão após complicações da insuficiência venosa mostraram-se dispostos a aceitar o desconforto oriundo desse dispositivo para reduzir o risco de novas complicações no futuro. Expressou-se na relação face a face os benefícios da terapia compressiva, a experiência positiva com a meia elástica e a importância das informações individualizadas ao paciente, respeitando a singularidade de cada $\mathrm{um}^{13}$.

A experiência positiva obtida com o uso de meias elásticas de compressão, evidenciada pelo alívio dos principais sintomas da insuficiência venosa, permite que pessoas idosas desenvolvam ou mantenham a capacidade funcional que permite o bem-estar. O bem-estar é singular e permeado de aspirações subjetivas, incluindo sentimentos de realização, satisfação e felicidade 9 .

Com o aumento da longevidade, é fundamental que as equipes de saúde fomentem ações de promoção da saúde, como caminhadas e alimentação saudável, que podem contribuir para a saúde vascular. Além disso, devem atuar na prevenção de complicações da insuficiência venosa (úlcera varicosa, flebite, trombose), com o incentivo de cuidados à saúde que incluem o uso de meias elásticas de compressão. Tais ações poderão, em longo prazo, minimizar os fatores que interferem na capacidade funcional da população idosa, promovendo maior independência para as atividades diárias e qualidade de vida.

Nesse sentido, os profissionais de saúde, especialmente o enfermeiro, devem atentar na importância do acolhimento e acompanhamento desses idosos no nível da atenção primária à saúde. Esse nível de atenção tem a responsabilidade pela coordenação do cuidado na Rede de Atenção à Saúde, atuando na referência e contrarreferência aos serviços especializados ${ }^{14}$.

O fortalecimento do vínculo da atenção primária com o serviço de angiologia poderá alinhar os fluxos de contrarreferência, possibilitando a continuidade do tratamento e, consequentemente, o apoio de forma eficaz para o uso da terapia compressiva. $O$ vínculo se faz pela construção de relações estabelecidas no acolhimento, na responsabilização e na confiança mútua entre profissionais e idosos, e representa um recurso terapêutico que pode impactar a adesão às orientações realizadas pelos profissionais ${ }^{15}$.

\section{Adversidades que permeiam o uso de meias elásticas de compressão}

As pessoas idosas revelaram que calçar e descalçar as meias elásticas são fatores dificultadores relativos a essa modalidade de terapia compressiva. Os participantes mencionaram necessitar da ajuda de familiares para realizar esse cuidado.

"Na hora de calçar, a meia aperta, preciso de ajuda. Detesto a meia, para ser bem sincera, acho difícil de colocar."(E10)

"Minha filha é que tem que colocar a meia em mim, que eu não consigo colocar, ela é muito dura, eu não consigo calçar.” (E11)

A dificuldade de calçar e descalçar as meias elásticas também foi descrita em outros estudos ${ }^{16,17}$. Esse achado chama a atenção, uma vez que, ao receber a prescrição de uso de meia elástica de compressão, seria importante que o profissional de saúde fizesse uma avaliação da capacidade funcional do idoso com o objetivo de conhecer sua destreza para calçá-la e descalçá-la.

Sabe-se que o envelhecimento pode provocar perdas da capacidade visual, limitar o funcionamento das articulações, e doenças crônicas podem agravar essas capacidades. Essas são de grande importância para que o idoso tenha habilidade de calçar esse dispositivo compressivo. Desse modo, a sistematização da assistência de enfermagem pode auxiliar essa clientela e seus familiares.

A obtenção de dados abrangentes por meio do histórico de enfermagem e de testes de capacidade funcional pode ser útil para o enfermeiro traçar suas intervenções com vistas a auxiliar o idoso na atividade de calçar e descalçar a meia elástica de compressão, até mesmo indicando os calçadores de meias que facilitam sua colocação. Caso perceba que a pessoa idosa apresenta limitações funcionais que dificultem a utilização das meias, o profissional precisa procurar, em sua rede social, familiares ou cuidadores que possam assisti-la, com vistas à manutenção da terapia compressiva.

Pesquisa que relatou a percepção de pacientes cirúrgicos com média de idade de 63 anos sobre a prevenção de trombose mostrou que esses não viam clareza nas orientações sobre o uso de meias de compressão elástica, por isso, poucos referiram que continuariam a usá-las após a alta hospitalar e não estavam certos por quanto tempo conseguiriam usálas ${ }^{17}$. Esses dados corroboram a importância de propiciar informações básicas, porém abrangentes, sobre o uso adequado desse dispositivo.

Estudo de revisão sobre promoção da saúde, condição crônica e envelhecimento apontou que o enfermeiro tem papel importante ante o idoso e sua família, por desenvolver ações 
educativas que favorecem o convívio com a doença crônica. Destarte, esses profissionais precisam estar preparados para assistir o binômio familiar-idoso em condição crônica ${ }^{18}$. Faz-se necessário, também, o investimento em pesquisas tecnológicas a fim de melhorar o tipo de material usado na confecção de meias elásticas de compressão. Além disso, é necessário que os profissionais de saúde estejam cientes das opções disponíveis em termos de design, estrutura, cor e grau de compressão ${ }^{19}$, a fim de oferecer sugestões que facilitem o uso e a manutenção das meias elásticas.

$\mathrm{Na}$ Alemanha, estudo comparou o uso de meias convencionais com outra denominada SoftFit, composta por um fio de silicone na banda superior, projetado para mantê-la no lugar sem exercer pressão extra. Os resultados mostraram que esse tipo de meia foi bem tolerado pelos participantes, facilitando a adesão ao cuidado, uma vez que era fácil de calçar e descalçar, proporcionando a sensação de menos dor à compressão ${ }^{20}$. No Reino Unido, pesquisa que discutiu o uso de Neo-slip ${ }^{\oplus}$ - calçador de baixo atrito que ajuda na aplicação de meias de compressão - mostrou que esse recurso foi efetivamente adotado por um centro ortopédico, obtendo um feedback positivo tanto por parte da equipe de saúde quanto dos pacientes ${ }^{21}$.

Os participantes da presente pesquisa também citaram como fatores dificultadores a sensação de calor e o desconforto causados pela compressão dos membros inferiores.

"Quando eu comecei a usar a meia, me atrapalhava! Ela apertava muito e dava queimação nas pernas. Me lembro que falei com a doutora assim: “Ah não, isso não serve para usar." (E4)

"Com a meia, coça bastante a perna, por isso não gosto de usar." (E9)

“Com esse calorão todo, eu não uso a meia, eu não aguento, é muito calor. Parece que, no calor, a meia aperta mais."(E13)

Corrobora esses resultados estudo realizado na Turquia com adultos que receberam indicação de uso de meia elástica de compressão para controle de insuficiência venosa. Esses relataram a sensação de calor e desconforto com o uso desse dispositivo. Como alternativa para superar essas adversidades e elevar a adesão à terapia compressiva, os autores destacaram a relevância do papel dos enfermeiros na conscientização sobre os benefícios gerados pelo uso regular das meias elásticas associada a mudanças no estilo de vida ${ }^{22}$.
Na presente investigação, os entrevistados consideraram que o uso de meias elásticas de compressão dificulta a realização das tarefas domésticas e influencia no atraso do deslocamento para o trabalho, devido ao tempo gasto para calçá-las.

"Eu detesto usar a meia, minha experiência é ruim, tira a liberdade para fazer uma limpeza na casa, porque, estando de meia, preciso colocar uma bota. É cansativo ficar com as meias, então muita coisa eu deixo de fazer por conta de estar de meia." (E2)

“O problema é tomar banho. Então, tomo banho e deito um pouco, uns 15 minutos, com a perna para cima para então colocar a meia. Isso me atrasa um pouco no trabalho."(E13)

Estudo fenomenológico destacou a dificuldade de uso da terapia compressiva prescrita devido às questões do cotidiano feminino, em especial quando as mulheres eram donas de casa. As participantes reportavam dificuldades relacionadas às necessidades elementares do cotidiano, como realizar a própria higiene e usar vestuário e calçados que necessitavam ser adaptados para atender ao cuidado exigido pela terapia compressiva $^{16}$.

Se, por um lado, o uso de meia elástica de compressão pode prevenir, em longo prazo, as complicações da insuficiência venosa e contribuir para a manutenção da capacidade funcional do idoso, principalmente as relacionadas à mobilidade, por outro, esse público percebe que a meia elástica interfere nas atividades cotidianas, dificultando sua realização - higiene, tarefas domésticas e laborais. Entretanto, o profissional de saúde deve ponderar com essa clientela os riscos de não manter o uso da terapia compressiva e discutir estratégias com os idosos que viabilizem concatenar o uso da meia com as atividades cotidianas.

A dificuldade de manter o uso de meia elástica de compressão devido ao custo elevado desse dispositivo foi referida pelos entrevistados.

“Tive muita dificuldade de comprar a meia por causa do preço. Comprava em bazar. Quando tiro a meia para lavar, eu só tenho ela, fico sem; preciso comprar outra." (E8)

“Cinco anos atrás, era $\mathrm{R} \$ 60,00$, achei muito caro, por isso que não comprei. Aí minha sobrinha comprou para mim e gostei." (E12). 
Estudo desenvolvido nos Países Baixos ressaltou que o custo elevado das meias elásticas de compressão não influenciou as preferências dos pacientes, pois o dispositivo e a assistência domiciliar para a aplicação da meia são subsidiados naquele país ${ }^{13}$. Tal programa poderia ser adotado no Brasil, considerando as condições socioeconômicas limitadas dos participantes do presente estudo, o que facilitaria a aquisição do dispositivo, assim como incentivaria seu uso.

\section{Uso de meia elástica de compressão em longo prazo}

Apesar das adversidades apontadas pelos participantes sobre o uso de meias elásticas de compressão, esses apresentaram a expectativa de usar esse dispositivo em longo prazo, com o objetivo de controlar os principais sintomas da condição crônica que o requer, como dor nos membros inferiores, varizes e recidiva de úlcera varicosa, com vistas a manter o desempenho das atividades cotidianas.

"O cuidado e a meia vão me acompanhar até eu morrer. Se eu não usar a meia, está arriscado a ferida abrir outra vez. [...] muita coisa deixava de fazer. Quero sair à noite, andar, passear, viajar, ir na igreja, capinar meu quintal." (E1)

"Ah, vou ter que usar a meia o resto da vida. É um sonho parar de usar a meia, nunca vou parar, porque eu sei que, se eu parar, a ferida pode abrir. Eu estou usando a meia com frequência, para eu continuar resolvendo minhas coisas: lavar, passar, cozinhar, sair de carro [...] sem meia, aí o negócio aperta, tenho que usar a meia mesmo.” (E2)

"Eu pretendo usar a meia, para ver se não sinto dor, se não estoura mais veias do que já tenho [...] com a meia eu vou andar o dia inteiro sem dor nas pernas." (E6)

"Não sei, vou usando a meia e vou vendo, espero que melhore. As varizes são difíceis de sarar, não sara. Com a meia fico sem dor nas pernas, posso andar e fazer minhas coisas." (E7)

Salienta-se que a utilização de meias elásticas de compressão nem sempre é fácil para o idoso, sobretudo aquelas de alta compressão, o que pode impactar a continuidade da terapia compressiva em longo prazo. Para que a pessoa idosa mantenha a continuidade do uso da meia elástica, faz-se necessário que os profissionais de saúde adequem o nível de compressão do dispositivo à capacidade do idoso de suportá-lo. A literatura aponta que é preferível usar a terapia compressiva com baixo grau de compressão do que deixar de usá-la .

Na Turquia, investigação com 219 pessoas avaliou o nível de conforto e eficácia de meias elásticas de compressão de três diferentes graduações de compressão e comprimento. Os resultados mostraram que, quanto ao conforto, $79,5 \%$ das pessoas se encontravam no grupo III - pressão moderada, na altura do joelho - e 52,1\% do grupo II - baixa pressão, na altura da coxa - declararam que sentem desconforto durante o uso das meias. A maioria das pessoas que utilizou a meia de baixa pressão, na altura do joelho (grupo I), relatou alto grau de satisfação e não referiu desconforto ${ }^{23}$.

A fim de garantir a intencionalidade dos participantes de continuar o uso das meias elásticas de compressão em longo prazo e manter a capacidade para realização de atividades diárias, ressalta-se a importância de os profissionais de saúde que atuam na atenção primária identificarem as dificuldades apresentadas pela pessoa idosa para usar a meia elástica de compressão e discutirem estratégias que facilitem seu uso em longo prazo, buscando subsídios na gestão dos serviços de saúde para viabilização de recursos necessários ao atendimento de idosos com essa condição crônica.

Ressalta-se que a concretização dessa intencionalidade está diretamente relacionada à valorização da relação intersubjetiva estabelecida entre o profissional e as pessoas idosas que necessitam usar esse dispositivo de compressão. Para a fenomenologia social de Alfred Schütz, o mundo cotidiano é cultural e intersubjetivo e, por isso mesmo que, em distintas situações biográficas, o homem compreende e é compreendido a partir das relações sociais que estabelece ${ }^{10}$. Nesse sentido, as interações estabelecidas entre o profissional de saúde e os idosos quanto à terapia compressiva podem ser mais significativas para ambos do que a tecnologia em si, no que tange ao controle da insuficiência venosa.

As relações do tipo face a face entre enfermeiro e pessoas idosas com insuficiência venosa que utilizam a meia elástica de compressão podem favorecer a exposição do acervo de conhecimentos ${ }^{10}$ de ambos, propiciando a construção de um plano de cuidados compartilhado que poderá resultar em uso mais frequente desse dispositivo de compressão. Nessa perspectiva, a construção desse plano de cuidados precisa contemplar a aprendizagem sobre a fisiopatologia dessa doença crônica, a corresponsabilização do cuidado e a valorização da 
experiência da pessoa com insuficiência venosa que usa as meias elásticas de compressão. Esse plano de cuidados deve ter como objetivo a manutenção da capacidade funcional do idoso, minimizando suas dificuldades e ajudando-o a enfrentá-las na execução das atividades cotidianas quando em uso de meia elástica de compressão.

Por se tratar de um estudo qualitativo realizado em um espaço geográfico e público específico, os resultados da presente investigação não podem ser generalizados. Contudo, somam evidências científicas sobre as questões envolvidas no uso de meias elásticas de compressão por pessoas idosas, o que poderá contribuir para impactar positivamente a assistência a esse público, assim como fomentar o ensino e a pesquisa em enfermagem.

A compreensão do cotidiano de idosos em relação ao uso de meias elásticas de compressão poderá subsidiar a atuação da equipe de saúde, em especial do enfermeiro estomaterapeuta, para valorizar as adversidades relacionadas ao uso desse dispositivo de modo a auxiliar esses idosos a ampliar sua autonomia e capacidade funcional comprometidas pela insuficiência venosa.

\section{CONCLUSÃO}

Segundo os participantes, o uso de meia elástica de compressão promove alívio dos principais sintomas da insuficiência venosa, permitindo o desempenho de atividades cotidianas. No entanto, apontaram adversidades que interferem na capacidade funcional desses idosos, como sensação de calor e desconforto nos membros inferiores e necessidade de ajuda para calçar e descalçar as meias. Os idosos esboçaram a expectativa de continuar usando a meia elástica em longo prazo com vistas a controlar a recidiva de úlcera varicosa.

Pesquisas que envolvem o desenvolvimento de novas tecnologias para aprimorar materiais para confecção das meias elásticas de compressão devem ser estimuladas, com vistas a facilitar o uso desse dispositivo e superar as adversidades cotidianas que permeiam seu uso, especialmente entre idosos.

\section{CONTRIBUIÇÃO DOS AUTORES}

Conceitualização, Silva MH e Ribeiro DK; Metodologia, Silva MH e Ribeiro DK; Investigação, Jesus R; Machado RET; Ribeiro DK e Silva MH; Redação - Primeira versão, Silva MH; Ribeiro DK; Jesus RR e Jesus MCP; Redação Revisão \& Edição, Silva MH; Jesus MCP e Merighi MAB; Aquisição de Financiamento, Ribeiro DK; Supervisão, Merighi MAB.

\section{REFERÊNCIAS}

1. Ouriel K. Central venous pathologies: treatments and economic impact. Methodist Debakey Cardiovasc J. 2018;14(3):166-72.

2. DePopas E, Brown M. Varicose veins and lower extremity venous insufficiency. Semin Intervent Radiol. 2018;35(1):5661. https://doi.org/10.1055\%2Fs-0038-1636522

3. Narbutt J, Bowszyc-Dmochowska M, Kapińska-Mrowiecka M, Kaszub A, Krasowska D, et al. Chronic venous insufficiency - pathogenesis, diagnosis and pharmacological treatment. Diagnostic and therapeutic recommendations of the Polish Dermatological Society. Part II. Dermatol Rev. 2018;105(4):486-97. https://doi.org/10.5114/dr.2018.78070

4. Wounds International. Principles of compression in venous disease: a practitioner's guide to treatment and prevention of venous leg ulcers [Internet]. Londres: Wounds International; 2013 [citado 30 jul 2019]. Disponível em: https://www. woundsinternational.com/resources/details/principlescompression-venous-disease-practitioners-guidetreatment-and-prevention-venous-leg-ulcers

5. Borges EL. Feridas: úlceras dos membros inferiores. 1. ed Rio de Janeiro: Guanabara Koogan; 2012.
6. Chwała M, Szczeklik W, Szczeklik M, Aleksiejew-Kleszczyński T, Jagielska-Chwała M. Varicose veins of lower extremities, hemodynamics and treatment methods. Adv Clin Exp Med. 2015;24(1):5-14. https://doi.org/10.17219/ acem/31880

7. Melo BV, Tojal PGMD, Leal FJ, Couto RC. Quality of life in chronic venous patients who do or do not wear compressive stockings. J Vasc Bras. 2015;14(1):62-7. https:// doi.org/10.1590/1677-5449.20140070

8. Silva CSO, Barbosa MMS, Pinho L, Figueiredo MFS, Amaral CO, Cunha FO, et al. Family health strategy: relevance to the functional capacity of older people. Rev Bras Enferm. 2018;71(Suppl 2):740-6. https://doi.org/10.1590/00347167-2017-0078

9. World Health Organization. World report on ageing and health [Internet]. Genebra: WHO; 2015. [citado 30 jul 2019]. Disponível em: http://apps.who.int/iris/ bitstream/10665/186463/1/9789240694811_eng. pdf?ua $=1$

10. Alfred Schutz. Sobre fenomenologia e relações sociais. Trad. Wagner HTR. Petrópolis: Vozes; 2012. 
11. Minayo MCS. Amostragem e saturação em pesquisa qualitativa: consensos e controvérsias. RPQ. 2017;5(7):1-12.

12. SilvaMH,Jesus MCP, Tavares RE, CaldeiraEAC, OliveiraDM, Merighi MAB. Experience of adults and older people with adherence to venous ulcer care. Rev Gaúcha Enferm. 2019;40:e20180024. https://doi.org/10.1590/1983-1447.2019.20180024

13. Bouman $A C$, Ten Cate-Hoek AJ, Dirksen CD, Joore MA. Eliciting patients' preferences for elastic compression stocking therapy after deep vein thrombosis: potential for improving compliance. J Thromb Haemost. 2016;14(3):5107. https://doi.org/10.1111/jth.13228

14. Ministério da Saúde (BR). Portaria $n^{\circ}$ 2.436, de 21 de setembro de 2017 [Internet]. Brasília, DF: Ministério da Saúde; 2017 [citado 30 jul 2019]. Disponível em: http://bvsms.saude.gov.br/bvs/saudelegis/gm/2017/ prt2436_22_09_2017.html

15. Santos RCA, Miranda FAN. Importância do vínculo entre profissional-usuário na Estratégia de Saúde da Família. Rev Enferm UFSM. 2016;6(3):350-9. https://doi. org/10.5902/2179769217313

16. Silva MHS, Jesus MCP, Merighi MAB, Oliveira DM. The selfcare experience of women living with chronic venous leg ulcer. ESTIMA, Braz J Enterostomal Ther. 2016;14(2):61-7. https://doi.org/10.5327/Z1806-3144201600020003

17. Apenteng PN, Fitzmaurice D, Litchfield I, Harrison S, Heneghan C, Ward A, et al. Patients' perceptions and experiences of the prevention of hospital-acquired thrombosis: a qualitative study. BMJ Open. 2016;6(12):e013839. https:// doi.org/10.1136/bmjopen-2016-013839

18. Valcarenghi RV, Lourenço LFL, Siewert JS, Alvarez AM. Nursing scientific production on health promotion, chronic condition, and aging. Rev Bras Enferm. 2015;68(4):705-12. https://doi.org/10.1590/0034-7167.2015680419i

19. Anderson I. Optimising concordance with compression hosiery in the community setting. Br J Community Nurs. 2015;20(2):6772. https://doi.org/10.12968/bjcn.2015.20.2.67

20. Münter C. Effect of a new compression garment on adherence: results of a patient satisfaction survey. Br J Nurs. 2015;11;24(Suppl 20):S44-9. https://doi.org/10.12968/ bjon.2015.24.Sup20.S44

21. Thomas N, Bennett N. Introducing a device to assist in the application ofanti-embolismstockings. BrJNurs.2017;26(9):51013. https://doi.org/10.12968/bjon.2017.26.9.510

22. Çoban PT, Dirimeşe E. Evaluation of quality of life after minimally invasive varicose vein treatment. Turk Gogus Kalp Dama. 2019;27(1):49-56. https://doi.org/10.5606/tgkdc. dergisi.2019.16867

23. Ayhan $H$, lyigun $E$, Ince $S$, Can MF, Hatipoglu S, Saglam M. A randomised clinical trial comparing the patient comfort and efficacy of three different graduated compression stockings in the prevention of postoperative deep vein thrombosis. J Clin Nurs. 2015;24(15-16):2247-57. https://doi.org/10.1111/ jocn.12866 